\title{
Convergence at first and second order of some approximations of stochastic integrals
}

\author{
Bérard Bergery Blandine, Vallois Pierre \\ IECN, Nancy-Université, CNRS, INRIA, Boulevard des Aiguillettes B.P. 239 \\ F-54506 Vandouvre lès Nancy
}

\begin{abstract}
We consider the convergence of the approximation schemes related to Itô's integral and quadratic variation, which have been developed in [8]. First, we prove that the convergence in the a.s. sense exists when the integrand is Hölder continuous and the integrator is a continuous semimartingale. Second, we investigate the second order convergence in the Brownian motion case.
\end{abstract}

Key words: stochastic integration by regularization, quadratic variation, first and second order convergence, stochastic Fubini's theorem 2000 MSC: 60F05, 60F17, 60G44, 60H05,60J65

\section{Introduction}

We consider a complete probability space $\left(\Omega, \mathcal{F}, \mathcal{F}_{t}, P\right)$, which satisfies the usual hypotheses. The notation (ucp) will stand for the convergence in probability, uniformly on the compact set in time.

1. Let $X$ be a real continuous $\left(\mathcal{F}_{t}\right)$-semimartingale. In the usual stochastic calculus, the quadratic variation and the stochastic integral with respect to $X$ play a central role. In [5], [6] and [7], Russo and Vallois extended these notions to continuous processes. Let us briefly recall their main definitions.

Definition 1.1 Let $X$ be a real-valued continuous process, $\left(\mathcal{F}_{t}\right)$-adapted, and $H$ be a locally integrable process. The forward integral $\int_{0}^{t} H d^{-} X$ is defined as

$$
\int_{0}^{t} H d^{-} X=\lim _{\epsilon \rightarrow 0}(u c p) \frac{1}{\epsilon} \int_{0}^{t} H_{u}\left(X_{u+\epsilon}-X_{u}\right) d u,
$$

if the limit exists. The quadratic variation is defined by

$$
[X]_{t}=\lim _{\epsilon \rightarrow 0}(u c p) \frac{1}{\epsilon} \int_{0}^{t}\left(X_{u+\epsilon}-X_{u}\right)^{2} d u
$$


if the limit exists.

In the article, $X$ will stand for a real-valued continuous $\left(\mathcal{F}_{t}\right)$-semimartingale and $\left(H_{t}\right)_{t \geqslant 0}$ for a $\left(\mathcal{F}_{t}\right)$-adapted process. If $H$ is continuous, then, according to Proposition 1.1 of [5], the limits in (1.1) exist and coincide with the usual objects. In order to work with adapted processes only, we change $u+\epsilon$ into $(u+\epsilon) \wedge t$ in the integrals. This change does not affect the limit by (3.3) of [8]. Consequently,

$$
\int_{0}^{t} H_{u} d X_{u}=\lim _{\epsilon \rightarrow 0}(u c p) \frac{1}{\epsilon} \int_{0}^{t} H_{u}\left(X_{(u+\epsilon) \wedge t}-X_{u}\right) d u,
$$

and

$$
<X>_{t}=\lim _{\epsilon \rightarrow 0}(u c p) \frac{1}{\epsilon} \int_{0}^{t}\left(X_{(u+\epsilon) \wedge t}-X_{u}\right)^{2} d u
$$

where $\int_{0}^{t} H_{u} d X_{u}$ is the usual stochastic integral and $\langle X\rangle$ is the usual quadratic variation.

2. First, we determine sufficient conditions under which the convergences in (1.1) and (1.2) hold in the almost sure sense. Let us mention that some results in this direction have been obtained in [2].

We say that a process $Y$ is locally Hölder continuous if, for all $T>0$, there exist $\left.\left.\alpha^{\prime} \in\right] 0,1\right]$ and $C_{T} \in L^{2}(\Omega)$ such that

$$
\left|Y_{s}-Y_{u}\right| \leqslant C_{T}|u-s|^{\alpha^{\prime}} \quad \forall u, s \in[0, T] \text {, a.s. }
$$

Our first result related to stochastic integral is the following.

Theorem 1.2 If $\left(H_{t}\right)_{t \geqslant 0}$ is adapted and locally Hölder continuous, then

$$
\lim _{\epsilon \rightarrow 0} \frac{1}{\epsilon} \int_{0}^{t} H_{u}\left(X_{(u+\epsilon) \wedge t}-X_{u}\right) d u=\int_{0}^{t} H_{u} d X_{u}
$$

in the sense of almost sure convergence, uniformly on the compact sets in time.

Proposition 1.3 If $X$ is locally Hölder continuous, then

$$
\lim _{\epsilon \rightarrow 0} \frac{1}{\epsilon} \int_{0}^{t}\left(X_{(u+\epsilon) \wedge t}-X_{u}\right)^{2} d u=<X>_{t},
$$

in the sense of almost sure convergence, uniformly on the compact sets in time. Moreover, if $H$ is a continuous process,

$$
\lim _{\epsilon \rightarrow 0} \frac{1}{\epsilon} \int_{0}^{t} H_{u}\left(X_{(u+\epsilon) \wedge t}-X_{u}\right)^{2} d u=\int_{0}^{t} H_{u} d<X>_{u},
$$

in the sense of almost sure convergence. 
3. Let us now consider the case where $X$ is the standard Brownian motion $B$. Since $B$ is a locally Hölder continuous martingale, the conditions in Theorem 1.2 and Proposition 1.3 are fulfilled. Then, it seems natural to determine the rate of convergence in (1.3) and (1.4), i.e. a second order convergence. Note that in [2], some related results have been proven.

Let us consider

$$
\begin{aligned}
\Delta_{\epsilon}(H, t) & =\frac{1}{\sqrt{\epsilon}}\left[\frac{1}{\epsilon} \int_{0}^{t} H_{u}\left(B_{(u+\epsilon) \wedge t}-B_{u}\right) d u-\int_{0}^{t} H_{u} d B_{u}\right], \\
\Delta_{\epsilon}^{(2)}(H, t) & =\frac{1}{\sqrt{\epsilon}}\left[\frac{1}{\epsilon} \int_{0}^{t} H_{u}\left(B_{(u+\epsilon) \wedge t}-B_{u}\right)^{2} d u-\int_{0}^{t} H_{u} d u\right],
\end{aligned}
$$

where $H$ is a progressively measurable process such that $\int_{0}^{t} H_{s}^{2} d s<\infty$, for every $t>0$.

We begin with $H_{t}=B_{t}$. In this case, we have:

$$
\Delta_{\epsilon}(H, t)=W_{\epsilon}(t)+R_{\epsilon}(t)
$$

where

$$
W_{\epsilon}(t)=\int_{0}^{t} G_{\epsilon}(u) d B_{u}, \quad G_{\epsilon}(u)=\frac{1}{\epsilon \sqrt{\epsilon}} \int_{(u-\epsilon)^{+}}^{u}\left(B_{u}-B_{s}\right) d s,
$$

and

$$
R_{\epsilon}(t)=\frac{1}{\sqrt{\epsilon}} \int_{0}^{t}\left(\frac{1}{\epsilon} \int_{0}^{u} B_{s} d s-\frac{u-\epsilon}{\epsilon} B_{u}\right) d B_{u} .
$$

It is easy to verify that $R_{\epsilon}(t) \rightarrow 0$, as $\epsilon \rightarrow 0$, in $L^{2}(\Omega)$, and therefore does not contribute to the limit.

Theorem $1.4\left(W_{\epsilon}(t), B_{t}\right)_{t \geqslant 0}$ converges in distribution to $\left(\sigma W_{t}, B_{t}\right)_{t \geqslant 0}$, as $\epsilon \rightarrow$ 0 , where $W$ is a standard Brownian motion, independent from $B$, and $\sigma^{2}=\frac{1}{3}$.

We now investigate the convergence of $\left(\Delta_{\epsilon}(H, t)\right)_{t \geqslant 0}$. We restrict ourselves to processes $H$ of the type $H_{t}=H_{0}+M_{t}+V_{t}$ where

1. $H_{0}$ is $\mathcal{F}_{0}$-measurable,

2. $M_{t}$ is a Brownian martingale, i.e. $M_{t}=\int_{0}^{t} \Lambda_{s} d B_{s}$, where $\left(\Lambda_{t}\right)$ is progressively measurable and satisfies $\int_{0}^{t} \Lambda_{s}^{2} d s<\infty$,

3. $V$ is a continuous process, which is Hölder continuous with order $\alpha>1 / 2$, vanishing at time 0 .

Note that if $V_{t}=\int_{0}^{t} \Phi_{s} d s$, where $\left(\Phi_{t}\right)_{t \geqslant 0}$ is adapted and locally bounded, then (3) holds with $\alpha=1$ and $H_{t}$ is a semimartingale.

Using a functional theorem of convergence (Proposition 3.2 and Theorem 5.1 in [3]) and Theorem 1.4, we obtain the following result. 
Theorem 1.5 1. For any $0<t_{1}<\cdots<t_{n}$, the random vector $\left(\Delta_{\epsilon}\left(H_{0}, t_{1}\right)\right.$, $\left.\ldots, \Delta_{\epsilon}\left(H_{0}, t_{n}\right)\right)$ converges in law to $\left(\sigma H_{0} N_{0}, \ldots, \sigma H_{0} N_{0}\right)$, where $N_{0}$ is a standard Gaussian r.v, independent from $\mathcal{F}_{0}$.

2. If $V$ is a process which is locally Hölder continuous of order $\alpha>\frac{1}{2}$, then $\Delta_{\epsilon}(V, t)$ converges to 0 in $L^{2}(\Omega)$ as $\epsilon \rightarrow 0$, uniformly on the compact set in time.

3. If $M_{t}=\int_{0}^{t} \Lambda_{s} d B_{s}$, where $\Lambda_{s}=f\left(B_{u}, u \leqslant s\right)$ is a continuous function of the trajectory $\left(B_{u}, u \leqslant s\right)$ such that $t \rightarrow \Lambda_{t}$ is a continuous map from $\mathbb{R}^{+}$to $L^{2}(\Omega)$, then the process $\left(\Delta_{\epsilon}(M, t)\right)_{t \geqslant 0}$ converges in distribution to $\left(\sigma \int_{0}^{t} \Lambda_{u} d W_{u}\right)_{t \geqslant 0}$ as $\epsilon \rightarrow 0$.

4. If $H_{0}=0, M$ and $V$ are as in point $(2)-(3)$, then $\left(\Delta_{\epsilon}(H, t)\right)_{t \geqslant 0}$ converges in law to $\left(\sigma \int_{0}^{t} \Lambda_{u} d W_{u}\right)_{t \geqslant 0}$ as $\epsilon \rightarrow 0$.

The conditions of Theorem 1.5 related to the process $H$ are likely too strong. In particular, the continuity of $t \rightarrow H_{t}$ and the fact that $H$ is a semimartingale are not needed. Indeed, there exist adapted stepwise processes $H$ so that $\Delta_{\epsilon}(H, t)$ converges in distribution as $\epsilon \rightarrow 0$, for any $t>0$. More precisely, we have the following result.

Theorem 1.6 Let $\left(a_{i}\right)_{i \in \mathbb{N}}$ be an increasing sequence of real numbers which satisfies $a_{0}=0$ and $a_{n} \rightarrow \infty$. Let $h,\left(h_{i}\right)_{i \in \mathbb{N}}$ be r.v.'s such that $h_{i}$ is $\mathcal{F}_{a_{i}}$ measurable, $h$ is $\mathcal{F}_{0}$-measurable and $\sup _{i \in \mathbb{N}}\left\|h_{i}\right\|_{\infty}<\infty$. Let $H$ be the adapted and stepwise process:

$$
H_{t}=h \mathbb{1}_{\{t=0\}}+\sum_{i \geqslant 0} h_{i} \mathbb{1}_{\left.\left.\{t \in] a_{i}, a_{i+1}\right]\right\}} .
$$

Then,

1. $\frac{1}{\epsilon} \int_{0}^{t} H_{s}\left(B_{(s+\epsilon) \wedge t}-B_{s}\right) d s$ converges almost surely to $\int_{0}^{t} H_{s} d B_{s}$, uniformly on the compact set in time, as $\epsilon \rightarrow 0$.

2. There exists a sequence of i.i.d. r.v's $\left(N_{i}\right)_{i \in \mathbb{N}}$ with Gaussian law $\mathcal{N}(0,1)$, independent from $B$ such that the r.v. $\Delta_{\epsilon}(H, t)$ converges in law to

$$
\frac{h_{0}}{\sqrt{3}} N_{0}+\sum_{i \geqslant 1} \frac{\left(h_{i}-h_{i-1}\right)}{\sqrt{3}} N_{i} \mathbb{I}_{\left\{t \leqslant a_{i+1}\right\}},
$$

as $\epsilon \rightarrow 0$, for fixed time $t$.

A proof of Theorem 1.6 can be found in Section 6.3 of [1].

4. Let us finally present our second order result of convergence related to quadratic variation. 
Proposition 1.7 Let $H_{s}=f\left(B_{u}, u \leqslant s\right)$ be a continuous function of the trajectory $\left(B_{u}, u \leqslant s\right)$ such that $s \rightarrow H_{s}$ is locally Hölder continuous. Then, $\left(\Delta_{\epsilon}^{(2)}(H, t)\right)_{t \geqslant 0}$ converges in distribution to $\left(\sigma \int_{0}^{t} H_{u} d W_{u}\right)_{t \geqslant 0}$, as $\epsilon \rightarrow 0$.

5. Let us briefly detail the organization of the paper. Section 2 contains the proofs of the almost convergence results, i.e. Theorem 1.2 and Proposition 1.3. Then, the proof of Theorem 1.4 (resp. Proposition 1.7 and Theorem 1.5) is (resp. are) given in Section 3 (resp. Section 4).

In the calculations, $C$ will stand for a generic constant (random or not). If $C$ is random, then $C \in L^{2}(\Omega)$. We will use several times a stochastic version of Fubini's Theorem, which can be found in Section IV.5 of [4].

\section{Proof of Theorem 1.2 and Proposition 1.3}

We begin with the proof of Theorem 1.2 in Points $\mathbf{1 - 4}$ below. Then, we deduce Proposition 1.3 from Theorem 1.2 in Point 5.

1. Let $T>0$. We suppose that $\left(H_{t}\right)_{t \geqslant 0}$ is locally Hölder continuous of order $\alpha^{\prime}$ and we study the almost sure convergence of

$$
I_{\epsilon}(t):=\frac{1}{\epsilon} \int_{0}^{t} H_{u}\left(X_{(u+\epsilon) \wedge t}-X_{u}\right) d u \text { to } I(t):=\int_{0}^{t} H_{u} d X_{u}
$$

as $\epsilon \rightarrow 0$, uniformly on $t \in[0, T]$.

By stopping, we can suppose that $\left(X_{t}\right)_{0 \leqslant t \leqslant T}$ and $<X>_{T}$ are bounded by a constant, and there exists a constant $C>0$ such that

$$
\left.\forall u, s \in[0, T], \quad\left|H_{s}-H_{u}\right| \leqslant C|u-s|^{\alpha}, \quad \text { for some } \alpha \in\right] 0, \alpha^{\prime}[\text {. }
$$

Let $X=X_{0}+M+V$ be the canonical decomposition of $X$, where $M$ is a continuous local martingale and $V$ is an adapted process with finite variation. It is clear that $I_{\epsilon}(t)-I(t)$ can be decomposed as

$$
\begin{aligned}
I_{\epsilon}(t)-I(t)= & \left(\frac{1}{\epsilon} \int_{0}^{t} H_{u}\left(M_{(u+\epsilon) \wedge t}-M_{u}\right) d u-\int_{0}^{t} H_{u} d X_{u}\right) \\
& +\left(\frac{1}{\epsilon} \int_{0}^{t} H_{u}\left(M_{(u+\epsilon) \wedge t}-M_{u}\right) d u-\int_{0}^{t} H_{u} d X_{u}\right) .
\end{aligned}
$$

Theorem 1.2 will be proved as soon as $I_{\epsilon}(t)-I(t)$ converges to 0 , in the case where $X$ is either a continuous local martingale or a continuous finite variation process.

We deal with the finite variation case in Point 2. As for the martingale case, the study is divided in two steps: 
1. First, we prove that there is a sequence $\left(\epsilon_{n}\right)_{n \in \mathbb{N}}$ such that $I_{\epsilon_{n}}(t)$ converges almost surely to $I(t)$ and $\epsilon_{n} \rightarrow 0$ (see Point 3 below).

2. Second, we show that $I_{\epsilon}(t)$ converges almost surely to 0 , uniformly for $t \in[0, T]$ (see Point 4 below).

2. Suppose that $X$ has a finite variation, writing $X_{(u+\epsilon) \wedge t}-X_{u}=\int_{(u+\epsilon) \wedge t}^{u} d X_{s}$ and using Fubini's theorem yield to:

$$
\begin{aligned}
I_{\epsilon}(t)-I(t) & =\int_{0}^{t}\left(\frac{1}{\epsilon} \int_{(s-\epsilon)^{+}}^{s} H_{u} d u-H_{s}\right) d X_{s}, \\
& =\int_{0}^{t}\left(\frac{1}{\epsilon} \int_{(s-\epsilon)^{+}}^{s}\left(H_{u}-H_{s}\right) d u\right) d X_{s}-\int_{0}^{\epsilon} \frac{\epsilon-s}{\epsilon} H_{s} d X_{s} .
\end{aligned}
$$

Using the Hölder property (2.1) (in the first integral) and the fact that $H$ is bounded by a constant (in the second integral), we have for all $t \in[0, T]$ :

$$
\begin{aligned}
\left|I_{\epsilon}(t)-I(t)\right| & \leqslant \int_{0}^{T}\left(\frac{1}{\epsilon} \int_{(s-\epsilon)^{+}}^{s} C|u-s|^{\alpha} d u\right) d|X|_{s}+\int_{0}^{\epsilon} \frac{\epsilon-s}{\epsilon} C d|X|_{s}, \\
& \leqslant C \epsilon^{\alpha}|X|_{T}+C\left(|X|_{\epsilon}-|X|_{0}\right) .
\end{aligned}
$$

Consequently, $I_{\epsilon}(t)-I(t)$ converges almost surely to 0 , as $\epsilon \rightarrow 0$, uniformly on any compact set in time.

3. In the two next points, $X$ is a continuous martingale. We proceed as in step 2 above: observing that $X_{(u+\epsilon) \wedge t}-X_{u}=\int_{(u+\epsilon) \wedge t}^{u} d X_{s}$ and using Fubini's stochastic theorem come to

$$
I_{\epsilon}(t)-I(t)=\int_{0}^{t}\left(\frac{1}{\epsilon} \int_{(s-\epsilon)^{+}}^{s} H_{u} d u-H_{s}\right) d X_{s}
$$

Thus, $\left(I_{\epsilon}(t)-I(t)\right)_{t \in[0, T]}$ is a continuous local martingale. Moreover, $E(<$ $\left.\left.I_{\epsilon}-I\right\rangle_{t}\right)$ is bounded since $H$ and $\langle X\rangle$ are bounded on $[0, T]$.

Let us introduce $p=\frac{2(1-\alpha)}{\alpha^{2}}+1$. This explicit expression of $p$ in terms of $\alpha$ will be used later at the end of Point 4. Burkholder-Davis-Gundy inequalities give:

$E\left(\sup _{t \in[0, T]}\left|I_{\epsilon}(t)-I(t)\right|^{p}\right) \leqslant c_{p} E\left[\left(\int_{0}^{T}\left(\frac{1}{\epsilon} \int_{(s-\epsilon)^{+}}^{s} H_{u} d u-H_{s}\right)^{2} d<X>_{s}\right)^{\frac{p}{2}}\right]$.

The Hölder property (2.1) implies that:

$$
\left|\frac{1}{\epsilon} \int_{(s-\epsilon)^{+}}^{s} H_{u} d u-H_{s}\right| \leqslant \frac{1}{\epsilon} \int_{(s-\epsilon)^{+}}^{s}\left|H_{u}-H_{s}\right| d u \leqslant C \epsilon^{\alpha} .
$$


Consequently,

$$
E\left(\sup _{t \in[0, T]}\left|I_{\epsilon}(t)-I(t)\right|^{p}\right) \leqslant C \epsilon^{\alpha p} E\left[<X>_{T}\right]^{\frac{p}{2}} \leqslant C \epsilon^{\alpha p} .
$$

Then, for any $\delta>0$, Markov inequality leads to :

$$
P\left(\sup _{t \in[0, T]}\left|I_{\epsilon}(t)-I(t)\right|>\delta\right) \leqslant \frac{C \epsilon^{\alpha p}}{\delta^{p}} .
$$

Let us now define $\left(\epsilon_{n}\right)_{n \in \mathbb{N}^{\star}}$ by $\epsilon_{n}=n^{-\frac{2}{p \alpha}}$ for all $n>0$. Replacing $\epsilon$ by $\epsilon_{n}$ in (2.2) comes to:

$$
P\left(\sup _{t \in[0, T]}\left|I_{\epsilon_{n}}(t)-I(t)\right|>\delta\right) \leqslant \frac{C}{\delta^{p}} n^{-2}
$$

Since $\sum_{n=1}^{\infty} n^{-2}<\infty$, the Borel-Cantelli lemma implies that:

$$
\lim _{n \rightarrow \infty} \sup _{t \in[0, T]}\left|I_{\epsilon_{n}}(t)-I(t)\right|=0, \quad \text { a.s. }
$$

4. For all $\epsilon \in] 0,1[$, let $n=n(\epsilon)$ denote the integer such that $\left.\epsilon \in] \epsilon_{n+1}, \epsilon_{n}\right]$. Then, we decompose $I_{\epsilon}(t)-I(t)$ as follows:

$$
I_{\epsilon}(t)-I(t)=\left(I_{\epsilon}(t)-I_{\epsilon_{n}}(t)\right)+\left(I_{\epsilon_{n}}(t)-I(t)\right) .
$$

(2.3) gives the almost sure convergence of $I_{\epsilon_{n}}(t)$ to $I(t)$, uniformly on $[0, T]$. Therefore, the a.s convergence of $I_{\epsilon}(t)-I(t)$ to 0 , uniformly on $[0, T]$, will be obtained as soon as $I_{\epsilon}(t)-I_{\epsilon_{n}}(t)$ goes to 0 , uniformly on $[0, T]$.

From the definition of $I_{\epsilon}(t)$, it is easy to deduce that we have:

$$
\begin{aligned}
I_{\epsilon}(t)-I_{\epsilon_{n}}(t)= & \frac{1}{\epsilon}\left(\int_{0}^{t} H_{u}\left(X_{(u+\epsilon) \wedge t} d u-\int_{0}^{t} H_{u} X_{\left(u+\epsilon_{n}\right) \wedge t}\right) d u\right) \\
& +\left(\frac{1}{\epsilon}-\frac{1}{\epsilon_{n}}\right)\left(\int_{0}^{t} H_{u}\left(X_{\left(u+\epsilon_{n}\right) \wedge t}-X_{u}\right) d u\right) .
\end{aligned}
$$

The changes of variable either $v=u+\epsilon$ or $v=u+\epsilon_{n}$ lead to

$$
\begin{aligned}
I_{\epsilon}(t)-I_{\epsilon_{n}}(t)= & \frac{1}{\epsilon} \int_{\epsilon}^{t}\left(H_{v-\epsilon}-H_{v-\epsilon_{n}}\right) X_{v} d v \\
& +\frac{\epsilon_{n}-\epsilon}{\epsilon \epsilon_{n}}\left(\int_{\epsilon_{n}}^{t}\left(H_{v-\epsilon_{n}}-H_{v}\right) X_{v} d v\right)+R_{\epsilon}(t),
\end{aligned}
$$

where we gather under the notation $R_{\epsilon}(t)$ all the remaining terms. Let us observe that $R_{\epsilon}(t)$ is the sum of terms which are of the form $\frac{1}{\epsilon} \int_{a}^{b} \ldots d v$ where 
$|a-b| \leqslant \epsilon_{n}-\epsilon$ or $\left(\frac{1}{\epsilon}-\frac{1}{\epsilon_{n}}\right) \int_{a}^{b} \ldots d v$ where $|a-b| \leqslant \epsilon_{n}$. Since $H$ and $X$ are bounded on $[0, T]$, we have

$$
\left|R_{\epsilon}(t)\right| \leqslant C \frac{\epsilon_{n}-\epsilon}{\epsilon} \quad \forall t \in[0, T] .
$$

By the Hölder property (2.1), we get

$$
\left|H_{v-\epsilon}-H_{v-\epsilon_{n}}\right| \leqslant C\left(\epsilon_{n}-\epsilon\right)^{\alpha}, \quad\left|H_{v-\epsilon_{n}}-H_{v}\right| \leqslant C \epsilon_{n}^{\alpha} .
$$

Since $X$ and $H$ are bounded, we can deduce from (2.4), (2.5) and (2.6) that:

$$
\left|I_{\epsilon}(t)-I_{\epsilon_{n}}(t)\right| \leqslant C \frac{\left(\epsilon_{n}-\epsilon\right)^{\alpha}}{\epsilon}+C \frac{\left(\epsilon_{n}-\epsilon\right) \epsilon_{n}^{\alpha}}{\epsilon \epsilon_{n}}+C \frac{\epsilon-\epsilon_{n}}{\epsilon}, \forall t \in[0, T] .
$$

Using the definition of $\epsilon_{n}$, we infer

$$
\frac{\epsilon_{n}-\epsilon}{\epsilon} \leqslant C n^{-1}, \frac{\left(\epsilon_{n}-\epsilon\right)^{\alpha}}{\epsilon} \leqslant C n^{\frac{2(1-\alpha)}{p \alpha}-\alpha}, \frac{\left(\epsilon_{n}-\epsilon\right) \epsilon_{n}^{\alpha}}{\epsilon \epsilon_{n}} \leqslant n^{-\frac{2}{p}-1+\frac{2}{p \alpha}} \leqslant n^{\frac{2(1-\alpha)}{p \alpha}-\alpha} .
$$

Note that $p=\frac{2(1-\alpha)}{\alpha^{2}}+1$ implies that $\frac{2(1-\alpha)}{p \alpha}-\alpha<0$. As a result, $I_{\epsilon}(t)-I_{\epsilon_{n}}(t)$ goes to 0 a.s, uniformly on $[0, T]$, as $\epsilon \rightarrow 0$.

5. In this item, it is supposed that $X$ is a semimartingale, locally Hölder continuous. It is clear that $\frac{1}{\epsilon} \int_{0}^{t}\left(X_{(u+\epsilon) \wedge t}-X_{u}\right)^{2} d u$ equals

$$
\frac{1}{\epsilon}\left[\int_{0}^{t} X_{(u+\epsilon) \wedge t}^{2} d u-\int_{0}^{t} X_{(u+\epsilon) \wedge t} X_{u} d u-\int_{0}^{t} X_{u}\left(X_{(u+\epsilon) \wedge t}-X_{u}\right) d u\right] .
$$

Thanks to the change of variable $v=u+\epsilon$ in the first integral, after easy calculations, we get

$$
\frac{1}{\epsilon} \int_{0}^{t}\left(X_{(u+\epsilon) \wedge t}-X_{u}\right)^{2} d u=X_{t}^{2}-\frac{1}{\epsilon} \int_{0}^{\epsilon} X_{v \wedge t}^{2} d v-\frac{2}{\epsilon} \int_{0}^{t} X_{u}\left(X_{(u+\epsilon) \wedge t}-X_{u}\right) d u .
$$

Since $X$ is continuous, $\frac{1}{\epsilon} \int_{0}^{\epsilon} X_{v \wedge t}^{2} d v$ tends to $X_{0}^{2}$ a.s, uniformly on $[0, T]$. According to Theorem 1.2, we have

$$
\lim _{\epsilon \rightarrow 0} \frac{1}{\epsilon} \int_{0}^{t}\left(X_{(u+\epsilon) \wedge t}-X_{u}\right)^{2} d u=X_{t}^{2}-X_{0}^{2}-2 \int_{0}^{t} X_{u} d X_{u}
$$

Itô 's formula implies that the right-hand side of the above identity equals to $<X>_{t}$.

Replacing $(u+\epsilon) \wedge t$ by $(u+\epsilon)^{+}$does not change the limit. Then, the measure $\frac{1}{\epsilon}\left(X_{(u+\epsilon)^{+}}-X_{u}\right)^{2} d u$ converges a.s. to the measure $d<X>_{u}$. It leads to the convergence a.s. of $\frac{1}{\epsilon} \int_{0}^{t} H_{u}\left(X_{(u+\epsilon) \wedge t}-X_{u}\right)^{2} d u$ to $\int_{0}^{t} H_{u} d<X>_{u}$, for $H$ continuous process. 


\section{Proof of Theorem 1.4}

Recall that $W_{\epsilon}(t)$ and $G_{\epsilon}(t)$ are defined by (1.7). We study the convergence in distribution of the two dimensional process $\left(W_{\epsilon}(t), B_{t}\right)$, as $\epsilon \rightarrow 0$.

First, we determine the limit in law of $W_{\epsilon}(t)$. In Point 1 we demonstrate preliminary results. Then, we prove the convergence of the moments of $W_{\epsilon}(t)$ in Point 2. By the method of moments, the convergence in law of $W_{\epsilon}(t)$ for a fixed time is proven in Point $\mathbf{3}$. We deduce the finite-dimensionnal convergence in Point 4. Finally, Kolmogorov criterion concludes the proof in Point 5. Then, we briefly sketch in Point 6 the proof of the joint convergence of $\left(W_{\epsilon}(t)\right)_{t \geqslant 0}$ and $\left(B_{t}\right)_{t \geqslant 0}$. The approach is close to the one of $\left(W_{\epsilon}(t)\right)_{t \geqslant 0}$.

1. We begin by calculating the moments of $W_{\epsilon}(t)$ and $G_{\epsilon}(u)$. We denote by $\underline{\underline{\mathcal{L}}}$ the equality in law.

Lemma 3.1 $E\left[\left|G_{\epsilon}(u)\right|^{2}\right]=\frac{(u \wedge \epsilon)^{3}}{\epsilon^{3}} \sigma^{2}$. Moreover, for all $k \in \mathbb{N}$, there exists a constant $m_{k}$ such that $E\left[\left|G_{\epsilon}(u)\right|^{k}\right] \leqslant m_{k}, \forall u \geqslant 0, \epsilon>0$.

Proof. First, we apply the change of variable $s=u-(u \wedge \epsilon) r$ in (1.7). Then, using the identity $\left(B_{u}-B_{u-v} ; 0 \leqslant v \leqslant u\right) \stackrel{\mathcal{L}}{=}\left(B_{v} ; 0 \leqslant v \leqslant u\right)$ and the scaling property of $B$, we get

$$
G_{\epsilon}(u) \stackrel{\mathcal{L}}{=} \frac{(u \wedge \epsilon) \sqrt{u \wedge \epsilon}}{\epsilon \sqrt{\epsilon}} \int_{0}^{1} B_{r} d r .
$$

Since $\int_{0}^{1} B_{r} d r \stackrel{\mathcal{L}}{=} \sigma N$, where $\sigma^{2}=1 / 3$ and $N$ is a standard gaussian r.v, we obtain

$$
E\left[\left|G_{\epsilon}(u)\right|^{k}\right]=\frac{(u \wedge \epsilon)^{\frac{3 k}{2}}}{\epsilon^{\frac{3 k}{2}}} \sigma^{k} E\left[|N|^{k}\right] .
$$

Taking $k=2$ gives $E\left[\left|G_{\epsilon}(u)\right|^{2}\right]=\frac{(t \wedge \epsilon)^{3}}{\epsilon^{3}} \sigma^{2}$. Using $u \wedge \epsilon \leqslant \epsilon$ and (3.1), we get $E\left[\left|G_{\epsilon}(u)\right|^{k}\right] \leqslant m_{k}$ with $m_{k}=\sigma^{k} E\left[|N|^{k}\right]$.

Lemma 3.2 For all $k \geqslant 2$, there exists a constant $C(k)$ such that

$$
\forall t \geqslant 0, \quad E\left[\left|W_{\epsilon}(t)\right|^{k}\right] \leqslant C(k) t^{\frac{k}{2}}
$$

Moreover, for $k=2$, we have

$$
E\left[\left(W_{\epsilon}(u)-W_{\epsilon}\left((u-\epsilon)^{+}\right)\right)^{2}\right] \leqslant \sigma^{2} \epsilon, \quad \forall u \geqslant 0 .
$$

Proof. The Burkhölder-Davis-Gundy inequality and (1.7) give

$$
E\left[\left|W_{\epsilon}(t)\right|^{k}\right] \leqslant c(k) E\left[\left(\int_{0}^{t}\left(G_{\epsilon}(u)\right)^{2} d u\right)^{\frac{k}{2}}\right] .
$$


Then, Jensen inequality implies:

$$
E\left[\left(\int_{0}^{t}\left(G_{\epsilon}(u)\right)^{2} d u\right)^{\frac{k}{2}}\right] \leqslant t^{\frac{k}{2}-1} E\left[\int_{0}^{t}\left|G_{\epsilon}(u)\right|^{k} d u\right] .
$$

Finaly, applying Lemma 3.1 comes to

$$
E\left[\left|W_{\epsilon}(t)\right|^{k}\right] \leqslant c(k) m_{k} t^{\frac{k}{2}}
$$

The case $k=2$ can be easily treated via (1.7) and Lemma 3.1:

$$
\begin{aligned}
E\left[\left(W_{\epsilon}(u)-W_{\epsilon}\left((u-\epsilon)^{+}\right)\right)^{2}\right] & =\int_{(u-\epsilon)^{+}}^{u} E\left[\left(G_{\epsilon}(v)\right)^{2}\right] d v \\
& =\int_{(u-\epsilon)^{+}}^{u} \frac{\sigma^{2}}{\epsilon^{3}} d v \leqslant \sigma^{2} \epsilon .
\end{aligned}
$$

2. Let us now study the convergence of the moments of $W_{\epsilon}(t)$.

\section{Proposition 3.3}

$$
\lim _{\epsilon \rightarrow 0} E\left[\left(W_{\epsilon}(t)\right)^{2 n}\right]=E\left[\left(\sigma W_{t}\right)^{2 n}\right], \quad \forall n \in \mathbb{N}, t \geqslant 0 .
$$

Proof. a) We prove Proposition 3.3 by induction on $n \geqslant 1$.

For $n=1$, from Lemma 3.1, we have:

$$
E\left[\left(W_{\epsilon}(t)\right)^{2}\right]=\int_{0}^{t} E\left[\left(G_{\epsilon}(u)\right)^{2}\right] d u=\int_{0}^{t} \sigma^{2} \frac{(u \wedge \epsilon)^{3}}{\epsilon^{3}} d u .
$$

Then, $E\left[\left(W_{\epsilon}(t)\right)^{2}\right]$ converges to $\sigma^{2} t=E\left[\left(\sigma W_{t}\right)^{2}\right]$.

Let us suppose that (3.2) holds. First, we apply Itô's formula to $\left(W_{\epsilon}(t)\right)^{2 n+2}$. Second, taking the expectation reduces to 0 the martingale part. Finally, we get

$$
E\left[\left(W_{\epsilon}(t)\right)^{2 n+2}\right]=\frac{(2 n+2)(2 n+1)}{2} \int_{0}^{t} E\left[\left(W_{\epsilon}(u)\right)^{2 n}\left(G_{\epsilon}(u)\right)^{2}\right] d u .
$$

b) We admit for a while that

$$
E\left[\left(W_{\epsilon}(u)\right)^{2 n}\left(G_{\epsilon}(u)\right)^{2}\right] \longrightarrow \sigma^{2} E\left[\left(\sigma W_{u}\right)^{2 n}\right], \quad \forall u \geqslant 0 .
$$

Using Cauchy-Schwarz inequality and Lemmas 3.1, 3.2 give:

$$
\begin{aligned}
E\left[\left(W_{\epsilon}(u)\right)^{2 n}\left(G_{\epsilon}(u)\right)^{2}\right] & \leqslant \sqrt{E\left[\left(W_{\epsilon}(u)\right)^{4 n}\right] E\left[\left(G_{\epsilon}(u)\right)^{4}\right]} \\
& \leqslant \sqrt{C(4 n) u^{2 n} m_{4}} \leqslant \sqrt{C(4 n) m_{4}} u^{n} .
\end{aligned}
$$


Consequently, we may apply Lebesgue's theorem to (3.3), we have

$$
\begin{aligned}
\lim _{\epsilon \rightarrow 0} E\left[\left(W_{\epsilon}(t)\right)^{2 n+2}\right] & =\frac{(2 n+2)(2 n+1)}{2} \sigma^{2} \int_{0}^{t} E\left[\left(\sigma W_{u}\right)^{2 n}\right] d u, \\
& =\frac{(2 n+2)(2 n+1)}{2} \sigma^{2 n+2} \int_{0}^{t} u^{n} \frac{(2 n) !}{n ! 2^{n}} d u, \\
& =\frac{(2 n+2) !}{(n+1) ! 2^{n+1}}(\sigma \sqrt{t})^{2 n+2}=E\left[\left(\sigma W_{t}\right)^{2 n+2}\right] .
\end{aligned}
$$

c) We have now to prove (3.4). If $u=0, E\left[\left(W_{\epsilon}(0)\right)^{2 n}\left(G_{\epsilon}(0)\right)^{2}\right]=0=$ $\sigma^{2} E\left[\left(\sigma W_{0}\right)^{2 n}\right]$. If $u>0$, it is clear that:

$$
E\left[\left(W_{\epsilon}(u)\right)^{2 n}\left(G_{\epsilon}(u)\right)^{2}\right]=E\left[\left(W_{\epsilon}\left((u-\epsilon)^{+}\right)\right)^{2 n}\left(G_{\epsilon}(u)\right)^{2}\right]+\xi_{\epsilon}(u),
$$

where

$$
\xi_{\epsilon}(u)=E\left[\left\{\left(W_{\epsilon}(u)\right)^{2 n}-\left(W_{\epsilon}\left((u-\epsilon)^{+}\right)\right)^{2 n}\right\}\left(G_{\epsilon}(u)\right)^{2}\right] .
$$

Since $G_{\epsilon}(u)$ is independent from $\mathcal{F}_{(u-\epsilon)^{+}}$, we have

$$
E\left[\left(W_{\epsilon}\left((u-\epsilon)^{+}\right)\right)^{2 n}\left(G_{\epsilon}(u)\right)^{2}\right]=E\left[\left(W_{\epsilon}\left((u-\epsilon)^{+}\right)\right)^{2 n}\right] E\left[\left(G_{\epsilon}(u)\right)^{2}\right] .
$$

Finally, plugging the identity above in (3.5) gives:

$$
E\left[\left(W_{\epsilon}(u)\right)^{2 n}\left(G_{\epsilon}(u)\right)^{2}\right]=E\left[\left(W_{\epsilon}(u)\right)^{2 n}\right] E\left[\left(G_{\epsilon}(u)\right)^{2}\right]+\xi_{\epsilon}(u)+\tilde{\xi}_{\epsilon}(u),
$$

where

$$
\tilde{\xi}_{\epsilon}(u)=E\left[\left(W_{\epsilon}\left((u-\epsilon)^{+}\right)\right)^{2 n}-\left(W_{\epsilon}(u)\right)^{2 n}\right] E\left[\left(G_{\epsilon}(u)\right)^{2}\right] .
$$

Lemma 3.1 implies that $E\left[\left(G_{\epsilon}(u)\right)^{2}\right]$ tends to $\sigma^{2}$ as $\epsilon \rightarrow 0$. The recurrence hypothesis implies that $E\left[\left(W_{\epsilon}(u)\right)^{2 n}\right]$ converges to $E\left[\left(\sigma W_{u}\right)^{2 n}\right]$ as $\epsilon \rightarrow 0$. It remains to prove that $\xi_{\epsilon}(u)$ and $\tilde{\xi}_{\epsilon}(u)$ tend to 0 to conclude the proof.

The identity $a^{2 n}-b^{2 n}=(a-b) \sum_{k=0}^{2 n-1} a^{k} b^{2 n-1-k}$ implies that $\xi_{\epsilon}(u)$ is equal to the sum $\sum_{k=0}^{2 n-1} S_{k}(\epsilon, u)$, where

$S_{k}(\epsilon, u)=E\left[\left(W_{\epsilon}(u)-W_{\epsilon}\left((u-\epsilon)^{+}\right)\right)\left(G_{\epsilon}(u)\right)^{2}\left(W_{\epsilon}(u)\right)^{k}\left(W_{\epsilon}\left((u-\epsilon)^{+}\right)\right)^{2 n-1-k}\right]$.

Applying four times the Cauchy-Schwarz inequality comes to:

$$
\begin{aligned}
\left|S_{k}(\epsilon, u)\right| \leqslant & {\left[E\left(W_{\epsilon}(u)-W_{\epsilon}\left((u-\epsilon)^{+}\right)\right)^{2}\right]^{\frac{1}{2}}\left[E\left(G_{\epsilon}(u)\right)^{8}\right]^{\frac{1}{4}} } \\
& \times\left[E\left(W_{\epsilon}(u)\right)^{8 k}\right]^{\frac{1}{8}}\left[E\left(W_{\epsilon}\left((u-\epsilon)^{+}\right)\right)^{16 n-8-8 k}\right]^{\frac{1}{8}} .
\end{aligned}
$$

Lemmas 3.1 and 3.2 lead to

$$
\left|S_{k}(\epsilon, u)\right| \leqslant C(k) T^{n-\frac{1}{2}} \sqrt{\epsilon}, \quad \forall u \in[0, T] .
$$

Consequently, $\xi_{\epsilon}(u)$ tends to 0 as $\epsilon \rightarrow 0$. Using the same method, it is easy to prove that $\tilde{\xi}_{\epsilon}(u)$ tends to 0 as $\epsilon \rightarrow 0$.

3. From Proposition 3.3, it easy to deduce the convergence in law of $W_{\epsilon}(t)$ (t being fixed). 
Proposition 3.4 For any fixed $t \geqslant 0, W_{\epsilon}(t)$ converges in law to $\sigma W_{t}$, as $\epsilon \rightarrow 0$.

Let us recall the method of moments.

Proposition 3.5 Let $X,\left(X_{n}\right)_{n \in \mathbb{N}}$ be r.v's such that $E\left(|X|^{k}\right)<\infty, E\left(\left|X_{n}\right|^{k}\right)<$ $\infty, \forall k, n \in \mathbb{N}$ and

$$
\varlimsup_{k \rightarrow \infty} \frac{\left[E\left(X^{2 k}\right)\right]^{\frac{1}{2 k}}}{2 k}<\infty .
$$

If for all $k \in \mathbb{N}, \lim _{n \rightarrow \infty} E\left(X_{n}^{k}\right)=E\left(X^{k}\right)$, then $X_{n}$ converges in law to $X$ as $n \rightarrow \infty$.

Proof of Proposition 3.4. Let $t \geqslant 0$ be a fixed time. The odd moments of $W_{\epsilon}(t)$ are null. By Proposition 3.3, the even moments of $W_{\epsilon}(t)$ tends to $\sigma W_{t}$. Since $\sigma W_{t}$ is a Gaussian r.v. with variance $\sigma \sqrt{t}$, it is easy to check that (3.6) holds. As a result, $W_{\epsilon}(t)$ converges in law to $\sigma W_{t}$.

4. Next, we prove the finite-dimensionnal convergence.

Proposition 3.6 Let $0<t_{1}<t_{2}<\cdots<t_{n}$. Then, $\left(W_{\epsilon}\left(t_{1}\right), \ldots, W_{\epsilon}\left(t_{n}\right)\right)$ converges in law to $\left(\sigma W_{t_{1}}, \ldots, \sigma W_{t_{n}}\right)$, as $\epsilon \rightarrow 0$.

Proof. We take $n=2$ for simplicity. We consider $0<t_{1}<t_{2}$ and $\epsilon \in$ ] $0, t_{1} \wedge\left(t_{2}-t_{1}\right)\left[\right.$. Since $t_{1}>\epsilon$, note that $(u-\epsilon)^{+}=u-\epsilon$ for $u \in\left[t_{1}, t_{2}\right]$. We begin with the decomposition:

$$
W_{\epsilon}\left(t_{2}\right)=W_{\epsilon}\left(t_{1}\right)+\frac{1}{\epsilon \sqrt{\epsilon}} \int_{t_{1}+\epsilon}^{t_{2}}\left(\int_{u-\epsilon}^{u}\left(B_{u}-B_{s}\right) d s\right) d B_{u}+R_{\epsilon}^{1}\left(t_{1}, t_{2}\right),
$$

where $R_{\epsilon}^{1}\left(t_{1}, t_{2}\right)=\frac{1}{\epsilon \sqrt{\epsilon}} \int_{t_{1}}^{t_{1}+\epsilon}\left(\int_{u-\epsilon}^{u}\left(B_{u}-B_{s}\right) d s\right) d B_{u}$. Let us note that $W_{\epsilon}\left(t_{1}\right)$ is independent from $\frac{1}{\epsilon \sqrt{\epsilon}} \int_{t_{1}+\epsilon}^{t_{2}}\left(\int_{u-\epsilon}^{u}\left(B_{u}-B_{s}\right) d s\right) d B_{u}$.

Let us introduce $B_{t}^{\prime}=B_{t+t_{1}}-B_{t_{1}}, t \geqslant 0$. $B^{\prime}$ is a standard Brownian motion. The changes of variables $u=t_{1}+v$ and $r=s-t_{1}$ in $\int_{t_{1}+\epsilon}^{t_{2}}\left(\int_{u-\epsilon}^{u}\left(B_{u}-B_{s}\right) d s\right) d B_{u}$ leads to

$$
W_{\epsilon}\left(t_{2}\right)=W_{\epsilon}\left(t_{1}\right)+\Theta_{\epsilon}\left(t_{1}, t_{2}\right)+R_{\epsilon}^{2}\left(t_{1}, t_{2}\right)+R_{\epsilon}^{1}\left(t_{1}, t_{2}\right),
$$

where

$$
\begin{aligned}
\Theta_{\epsilon}\left(t_{1}, t_{2}\right) & =\frac{1}{\epsilon \sqrt{\epsilon}} \int_{0}^{t_{2}-t_{1}}\left(\int_{(v-\epsilon)^{+}}^{v}\left(B_{v}^{\prime}-B_{r}^{\prime}\right) d r\right) d B_{v}^{\prime} \\
R_{\epsilon}^{2}\left(t_{1}, t_{2}\right) & =\frac{1}{\epsilon \sqrt{\epsilon}} \int_{0}^{\epsilon}\left(\int_{0}^{v}\left(B_{v}^{\prime}-B_{r}^{\prime}\right) d r\right) d B_{v}^{\prime} .
\end{aligned}
$$

Straightforward calculation shows that $E\left[\left(R_{\epsilon}^{1}\left(t_{1}, t_{2}\right)\right)^{2}\right]$ and $E\left[\left(R_{\epsilon}^{2}\left(t_{1}, t_{2}\right)\right)^{2}\right]$ are bounded by $C \epsilon$. Thus, $R_{\epsilon}^{1}\left(t_{1}, t_{2}\right)$ and $R_{\epsilon}^{1}\left(t_{1}, t_{2}\right)$ converge to 0 in $L^{2}(\Omega)$. 
Proposition 3.4 gives the convergence in law of $\Theta_{\epsilon}\left(t_{1}, t_{2}\right)$ to $\sigma\left(W_{t_{2}}-W_{t_{1}}\right)$ and the convergence in law of $W_{\epsilon}\left(t_{1}\right)$ to $\sigma W_{t_{1}}$, as $\epsilon \rightarrow 0$.

Since $W_{\epsilon}\left(t_{1}\right)$ and $\Theta_{\epsilon}\left(t_{1}, t_{2}\right)$ are independent, the decomposition (3.7) implies that $\left(W_{\epsilon}\left(t_{1}\right), W_{\epsilon}\left(t_{2}\right)-W_{\epsilon}\left(t_{1}\right)\right)$ converges in law to $\left(\sigma W_{t_{1}}, \sigma\left(W_{t_{2}}-W_{t_{1}}\right)\right)$, as $\epsilon \rightarrow 0$. Proposition 3.4 follows immediately.

5. We end the proof of the convergence in law of the process $\left(W_{\epsilon}(t)\right)_{t \geqslant 0}$ by showing that the family of the laws of $\left(W_{\epsilon}(t)\right)_{t \geqslant 0}$ is tight as $\left.\left.\epsilon \in\right] 0,1\right]$.

Lemma 3.7 There exists a constant $K$ such that

$$
E\left[\left|W_{\epsilon}(t)-W_{\epsilon}(s)\right|^{4}\right] \leqslant K|t-s|^{2}, \quad 0 \leqslant s \leqslant t, \epsilon>0 .
$$

Proof. Applying Burkhölder-Davis-Gundy inequality, we obtain:

$E\left[\left|W_{\epsilon}(t)-W_{\epsilon}(s)\right|^{4}\right] \leqslant c E\left[\left(\int_{s}^{t}\left(G_{\epsilon}(u)\right)^{2} d u\right)^{2}\right] \leqslant c(t-s) \int_{s}^{t} E\left[\left(G_{\epsilon}(u)\right)^{4}\right] d u$.

Using Lemma 3.1, we get $E\left[\left|W_{\epsilon}(t)-W_{\epsilon}(s)\right|^{4}\right] \leqslant c m_{4}(t-s)^{2}$ and ends the proof (see Kolmogorov Criterion in Section XIII-1 of [4]).

6. To prove the joint convergence of $\left(W_{\epsilon}(t), B_{t}\right)_{t \geqslant 0}$ to $\left(\sigma W_{t}, B_{t}\right)_{t \geqslant 0}$, we mimick the approach developed in Points 1-5 above.

6.a. Convergence $\left(W_{\epsilon}(t), B_{t}\right)$ to $\left(\sigma W_{t}, B_{t}\right), t$ being fixed.

First, we prove that

$$
\lim _{\epsilon \rightarrow 0} E\left(W_{\epsilon}^{p}(t) B_{t}^{q}\right)=E\left(\left(\sigma W_{t}\right)^{p} B_{t}^{q}\right), \quad p, q \in \mathbb{N} .
$$

Let us note that the limit is null when either $p$ or $q$ is odd.

Using Itô's formula, we get

$$
E\left[\left(W_{\epsilon}(t)\right)^{p} B_{t}^{q}\right]=\frac{p(p-1)}{2} \alpha_{1}(t, \epsilon)+\frac{q(q-1)}{2} \alpha_{2}(t, \epsilon)+p q \alpha_{3}(t, \epsilon),
$$

where

$$
\begin{aligned}
\alpha_{1}(t, \epsilon) & =\int_{0}^{t} E\left[\left(W_{\epsilon}(u)\right)^{p-2} B_{u}^{q}\left(G_{\epsilon}(u)\right)^{2}\right] d u, \\
\alpha_{2}(t, \epsilon) & =\int_{0}^{t} E\left[\left(W_{\epsilon}(u)\right)^{p} B_{u}^{q-2}\right] d u, \\
\alpha_{3}(t, \epsilon) & =\int_{0}^{t} E\left[\left(W_{\epsilon}(u)\right)^{p-1} B_{u}^{q-1} G_{\epsilon}(u)\right] d u .
\end{aligned}
$$

To demonstrate (3.8), we proceed by induction on $q$, then by induction on $p$, $q$ being fixed.

First, we apply (3.8) with $q-2$ instead of $q$, then we have directly:

$$
\lim _{\epsilon \rightarrow 0} \alpha_{2}(t, \epsilon)=\int_{0}^{t} E\left[\left(\sigma W_{u}\right)^{p}\right] E\left[B_{u}^{q-2}\right] d u .
$$


As for $\alpha_{1}(t, \epsilon)$, we write

$$
\begin{aligned}
\left(W_{\epsilon}(u)\right)^{p-2} & =\left(W_{\epsilon}(u)\right)^{p-2}-\left(W_{\epsilon}\left((u-\epsilon)^{+}\right)\right)^{p-2}+\left(W_{\epsilon}\left((u-\epsilon)^{+}\right)\right)^{p-2} \\
B_{u}^{q} & =B_{u}^{q}-B_{(u-\epsilon)^{+}}^{q}+B_{(u-\epsilon)^{+}}^{q}
\end{aligned}
$$

We proceed similarly with $\alpha_{3}(t, \epsilon)$. Reasoning as in Point $\mathbf{2}$ and using the two previous identities, we can prove:

$$
\lim _{\epsilon \rightarrow 0} \alpha_{1}(t, \epsilon)=\sigma^{2} \int_{0}^{t} E\left[\left(\sigma W_{u}\right)^{p-2}\right] E\left[B_{u}^{q}\right] d u \text { and } \lim _{\epsilon \rightarrow 0} \alpha_{3}(t, \epsilon)=0 .
$$

Consequently, when either $p$ or $q$ is odd, then $\lim _{\epsilon \rightarrow 0} \alpha_{i}(t, \epsilon)=0,(i=1,2)$ and therefore:

$$
\lim _{\epsilon \rightarrow 0} E\left(W_{\epsilon}^{p}(t) B_{t}^{q}\right)=0=E\left(\left(\sigma W_{t}\right)^{p} B_{t}^{q}\right) .
$$

It remains to determine the limit in the case where $p$ and $q$ are even. Let us denote $p=2 p^{\prime}$ and $q=2 q^{\prime}$. Then we have

$$
\begin{aligned}
\lim _{\epsilon \rightarrow 0} \alpha_{1}(t, \epsilon) & =\int_{0}^{t} \sigma^{2} \frac{(p-2) !}{2^{p^{\prime}-1}\left(p^{\prime}-1\right) !} u^{p^{\prime}-1} \sigma^{p-2} \frac{q !}{2^{q^{\prime}}\left(q^{\prime}\right) !} u^{q^{\prime}} d u \\
& =\frac{(p-2) ! q !}{2^{p^{\prime}+q^{\prime}-1}\left(p^{\prime}-1\right) !\left(q^{\prime}\right) !\left(p^{\prime}+q^{\prime}\right)} \sigma^{p} t^{p^{\prime}+q^{\prime}}, \\
\lim _{\epsilon \rightarrow 0} \alpha_{2}(t, \epsilon) & =\int_{0}^{t} \frac{p !}{2^{p^{\prime}}\left(p^{\prime}\right) !} \sigma^{p} u^{p^{\prime}} \frac{(q-2) !}{2^{q^{\prime}-1}\left(q^{\prime}-1\right) !} u^{q^{\prime}-1} d u \\
& =\frac{p !(q-2) !}{2^{p^{\prime}+q^{\prime}-1}\left(p^{\prime}\right) !\left(q^{\prime}-1\right) !\left(p^{\prime}+q^{\prime}\right)} \sigma^{p} t^{p^{\prime}+q^{\prime}} .
\end{aligned}
$$

Then, it is easy to deduce

$$
\lim _{\epsilon \rightarrow 0} E\left[\left(W_{\epsilon}(t)\right)^{p} B_{t}^{q}\right]=\frac{p !}{2^{p^{\prime}}\left(p^{\prime}\right) !} \sigma^{p} t^{p^{\prime}} \frac{q !}{2^{q^{\prime}}\left(q^{\prime}\right) !} t^{q^{\prime}}=E\left[\left(\sigma W_{t}\right)^{p}\right] E\left[B_{t}^{q}\right] .
$$

Next, we use a two dimensional version of the method of moments:

Proposition 3.8 Let $X, Y,\left(Y_{n}\right)_{n \in \mathbb{N}}\left(X_{n}\right)_{n \in \mathbb{N}}$ be r.v. whose moments are finite. Let us suppose that $X$ and $Y$ satisfy (3.6) and that $\forall p, q \in \mathbb{N}, \lim _{n \rightarrow \infty} E\left(X_{n}^{p} Y_{n}^{q}\right)$ $=E\left(X^{p} Y^{q}\right)$. Then, $\left(X_{n}, Y_{n}\right)$ converges in law to $(X, Y)$ as $n \rightarrow \infty$.

Since $W_{t}$ and $B_{t}$ are Gaussian r.v's, they both satisfy (3.6). Consequently, $\left(W_{\epsilon}(t), B_{t}\right)$ converges in law to $\left(\sigma W_{t}, B_{t}\right)$ as $\epsilon \rightarrow 0$.

6.b. Finite-dimensional convergence. Let $0<t_{1}<t_{2}$. We prove that $\left(W_{\epsilon}\left(t_{1}\right), W_{\epsilon}\left(t_{2}\right), B_{t_{1}}, B_{t_{2}}\right)$ converges in law to $\left(\sigma W_{t_{1}}, \sigma W_{t_{2}}, B_{t_{1}}, B_{t_{2}}\right)$. We apply decomposition (3.7) to $W_{\epsilon}\left(t_{2}\right)$.

By Point 6.a, $\left(W_{\epsilon}\left(t_{1}\right), B_{t_{1}}\right)$ converges in law to $\left(\sigma W_{t_{1}}, B_{t_{1}}\right)$ and $\left(\Theta_{\epsilon}\left(t_{1}, t_{2}\right), B_{t_{2}}-\right.$ $\left.B_{t_{1}}\right)$ converges to $\left(\sigma W_{t_{2}}-\sigma W_{t_{1}}, B_{t_{2}}-B_{t_{1}}\right)$. Since $\left(\Theta_{\epsilon}\left(t_{1}, t_{2}\right), B_{t_{2}}-B_{t_{1}}\right)$ is independent from $\left(W_{\epsilon}\left(t_{1}\right), B_{t_{1}}\right)$, we can conclude that $\left(W_{\epsilon}\left(t_{1}\right), W_{\epsilon}\left(t_{2}\right), B_{t_{1}}, B_{t_{2}}\right)$ converges in law to $\left(\sigma W_{t_{1}}, \sigma W_{t_{2}}, B_{t_{1}}, B_{t_{2}}\right)$. 


\section{Proofs of Theorem 1.5 and Proposition 1.7}

1. Convergence in distribution of a family of stochastic integrals with respect to $W_{\epsilon}$.

Recall that $W_{\epsilon}$ is a continuous martingale, which converges in distribution to $\sigma W$ as $\epsilon \rightarrow 0$. Then, by Proposition 3.2 of [3], $W_{\epsilon}$ satisfies the condition of uniform tightness.

Consequently, from Theorem 5.1 of [3], we can deduce that for any càdlàg predictable process $\Lambda$ such that $\left(\Lambda, W_{\epsilon}\right)$ converges in distribution to $(\Lambda, W)$, then the process $\left(\int_{0}^{t} \Lambda_{u} d W_{\epsilon}(u)\right)_{t \geqslant 0}$ converges in distribution to $\left(\sigma \int_{0}^{t} \Lambda_{u} d W_{u}\right)_{t \geqslant 0}$ as $\epsilon \rightarrow 0$.

2. Proof of Proposition 1.7. Recall that $\Delta_{\epsilon}^{(2)}(H, t)$ is defined by (1.6).

First, let us consider the case $H_{t}=1$ for all $t \geqslant 0$. Using Itô's formula, we obtain:

$$
\left(B_{(s+\epsilon) \wedge t}-B_{s}\right)^{2}=2 \int_{s}^{(s+\epsilon) \wedge t}\left(B_{u}-B_{s}\right) d B_{u}+(s+\epsilon) \wedge t-s .
$$

Reporting in $\Delta_{\epsilon}^{(2)}(t)$ and applying stochastic Fubini's theorem come to

$$
\Delta_{\epsilon}^{(2)}(1, t)=2 W_{\epsilon}(t)+\frac{1}{\sqrt{\epsilon}}\left(\int_{0}^{t} \frac{(s+\epsilon) \wedge t-s}{\epsilon} d s-t\right) .
$$

More generally, if we consider $H_{s}=f\left(B_{u}, u \leqslant s\right)$ a continuous function of the trajectory so that $t \rightarrow H_{t}$ is locally Hölder continuous, we can write a similar decomposition:

$$
\Delta_{\epsilon}^{(2)}(H, t)=2 \int_{0}^{t} H_{u} d W_{\epsilon}(u)+R_{\epsilon}(t)
$$

where

$R_{\epsilon}(t)=\frac{2}{\epsilon \sqrt{\epsilon}} \int_{0}^{t}\left[\int_{(u-\epsilon)^{+}}^{u}\left(H_{s}-H_{u}\right)\left(B_{u}-B_{s}\right) d s\right] d B_{u}+\frac{1}{\epsilon \sqrt{\epsilon}} \int_{(t-\epsilon)^{+}}^{t} H_{s}(t-s-\epsilon) d s$.

Since $s \rightarrow H_{s}$ is continuous, then a.s.

$$
\lim _{\epsilon \rightarrow 0} \sup _{t \in[0, T]}\left|\frac{1}{\epsilon \sqrt{\epsilon}} \int_{(t-\epsilon)^{+}}^{t} H_{s}(t-s-\epsilon) d s\right|=0 .
$$

Using the Hölder property of $H$ and Doob inequality, we easily obtain

$$
E\left[\sup _{t \in[0, T]}\left(\frac{2}{\epsilon \sqrt{\epsilon}} \int_{0}^{t}\left[\int_{(u-\epsilon)^{+}}^{u}\left(H_{s}-H_{u}\right)\left(B_{u}-B_{s}\right) d s\right] d B_{u}\right)^{2}\right] \leqslant C \epsilon^{2 \alpha} .
$$

Since $H_{s}=f\left(B_{u}, u \leqslant s\right)$ is a continuous function of the trajectory and $\left(W_{\epsilon}, B\right)$ converges in distribution to $(\sigma W, B)$ (cf. Theorem 1.4), applying Point 1 with $\Lambda=H$ leads to Proposition 1.7.

3. Proof of Point (2) of Theorem 1.5. Recall that $\Delta_{\epsilon}(H, t)$ is defined by (1.5). Since $V$ vanishes at time 0 , we prolong $V$ to $]-\infty,+\infty\left[\right.$, setting $V_{s}=0$ 
if $s \leqslant 0$. Using $B_{(s+\epsilon) \wedge t}-B_{s}=\int_{s}^{(s+\epsilon) \wedge t} d B_{u}$, then Fubini's stochastic theorem yields to

$$
\Delta_{\epsilon}(V, t)=\int_{0}^{t}\left(\frac{1}{\epsilon \sqrt{\epsilon}} \int_{u-\epsilon}^{u}\left(V_{s}-V_{u}\right) d s\right) d B_{u}
$$

Consequently,

$$
E\left(\sup _{t \in[0, T]}\left|\Delta_{\epsilon}(V, t)\right|^{2}\right) \leqslant 4 \int_{0}^{T} E\left(\frac{1}{\epsilon \sqrt{\epsilon}} \int_{u-\epsilon}^{u}\left(V_{s}-V_{u}\right) d s\right)^{2} d u .
$$

Let us bound the term in parenthesis. Since $V$ is Hölder continuous, we have

$$
\left|\frac{1}{\epsilon \sqrt{\epsilon}} \int_{u-\epsilon}^{u}\left(V_{s}-V_{u}\right) d s\right| \leqslant \frac{1}{\epsilon \sqrt{\epsilon}} \int_{u-\epsilon}^{u} C|s-u|^{\alpha} d s \leqslant C \epsilon^{\alpha-\frac{1}{2}} .
$$

Consequently,

$$
E\left(\sup _{t \in[0, T]}\left|\Delta_{\epsilon}(V, t)\right|^{2}\right) \leqslant C T \epsilon^{\alpha-\frac{1}{2}}
$$

Since $\alpha>\frac{1}{2}$, item (2) of Theorem 1.5 is proved.

\section{Proof of Point (3) of Theorem 1.5.}

Let $\Lambda_{s}=f\left(B_{u}, u \leqslant s\right)$ be a continuous function of the trajectory, such that $t \rightarrow \Lambda_{t}$ is a continuous map from $\mathbb{R}^{+}$to $L^{2}(\Omega)$. Suppose moreover that $M_{t}=$ $\int_{0}^{t} \Lambda_{u} d B_{u}$. Proceeding as in Point 3 of section 2, we have

$$
\Delta_{\epsilon}(M, t)=\int_{0}^{t} \frac{1}{\sqrt{\epsilon}}\left[\frac{1}{\epsilon} \int_{(u-\epsilon)^{+}}^{u} M_{s} d s-M_{u}\right] d B_{u} .
$$

Using the identity $M_{u}=\frac{u-(u-\epsilon)^{+}}{\epsilon} M_{u}+\frac{(\epsilon-u)^{+}}{\epsilon} M_{u}$ leads to $\Delta_{\epsilon}(M, t)=\Delta_{\epsilon}^{\prime}(M, t)-$ $R_{\epsilon}(t)$, where

$$
\Delta_{\epsilon}^{\prime}(M, t)=-\int_{0}^{t}\left(\frac{1}{\epsilon \sqrt{\epsilon}} \int_{(u-\epsilon)^{+}}^{u}\left(M_{u}-M_{s}\right) d s\right) d B_{u}
$$

and $R_{\epsilon}(t)=\int_{0}^{\epsilon} \frac{\epsilon-u}{\epsilon \sqrt{\epsilon}} M_{u} d B_{u}$. We claim that $R_{\epsilon}(t)$ is a remainder term. Indeed, Doob's inequality gives

$$
E\left[\sup _{t \in[0, T]} R_{\epsilon}(t)^{2}\right] \leqslant 4 \int_{0}^{\epsilon} \frac{(\epsilon-u)^{2}}{\epsilon^{3}} E\left[\int_{0}^{u} \Lambda_{v} d B_{v}\right]^{2} d u \leqslant \frac{4}{\epsilon} \int_{0}^{\epsilon} \int_{0}^{u} E\left[\Lambda_{v}^{2}\right] d v d u .
$$

Consequently, $R_{\epsilon}(t)$ does not contribute to the limit in law of $\Delta_{\epsilon}(M, t)$.

Using the identity $M_{u}-M_{s}=\Lambda_{u}\left(B_{u}-B_{s}\right)+\int_{s}^{u}\left(\Lambda_{r}-\Lambda_{u}\right) d B_{r}$, we decompose $\int_{(u-\epsilon)^{+}}^{u}\left(M_{u}-M_{s}\right) d s$ as

$$
\begin{aligned}
& \Lambda_{u} \int_{(u-\epsilon)^{+}}^{u}\left(B_{u}-B_{s}\right) d s+\int_{(u-\epsilon)^{+}}^{u}\left(\int_{s}^{u}\left(\Lambda_{r}-\Lambda_{u}\right) d B_{r}\right) d s \\
& =\Lambda_{u} \int_{(u-\epsilon)^{+}}^{u}\left(B_{u}-B_{s}\right) d s+\int_{(u-\epsilon)^{+}}^{u}\left(r-(u-\epsilon)^{+}\right)\left(\Lambda_{r}-\Lambda_{u}\right) d B_{r} .
\end{aligned}
$$


Consequently:

$$
\Delta_{\epsilon}^{\prime}(M, t)=-\int_{0}^{t} \Lambda_{u} d W_{\epsilon}(u)+R_{\epsilon}^{\prime}(t)
$$

where

$$
R_{\epsilon}^{\prime}(t)=-\frac{1}{\epsilon \sqrt{\epsilon}} \int_{0}^{t}\left(\int_{(u-\epsilon)^{+}}^{u}\left(r-(u-\epsilon)^{+}\right)\left(\Lambda_{r}-\Lambda_{u}\right) d B_{r}\right) d B_{u}
$$

It can be proved, as it is shown previously, that $R_{\epsilon}^{\prime}(t)$ goes to 0 in $L^{2}(\Omega)$ as $\epsilon \rightarrow 0$. The convergence in law of $\left(-\int_{0}^{t} \Lambda_{u} d W_{\epsilon}(u)\right)_{t \geqslant 0}$ to $\left(\sigma \int_{0}^{t} \Lambda_{u} d W_{u}\right)_{t \geqslant 0}$ is a direct consequence of item 1 above.

5. Proof of Point (1) of Theorem 1.5. We have, for a fixed $t$ and for all $\epsilon<t$ :

$$
\Delta_{\epsilon}\left(H_{0}, t\right)=\frac{H_{0}}{\sqrt{\epsilon}}\left[\frac{1}{\epsilon} \int_{0}^{t}\left(B_{(s+\epsilon) \wedge t}-B_{s}\right) d s-\int_{0}^{t} d B_{s}\right] .
$$

Since $B_{(s+\epsilon) \wedge t}-B_{s}=\int_{s}^{(s+\epsilon) \wedge t} d B_{u}$, using Fubini's Theorem allows to gather the integrals and we get

$$
\Delta_{\epsilon}\left(H_{0}, t\right)=\frac{H_{0}}{\sqrt{\epsilon}}\left[\int_{0}^{t} \frac{u-\epsilon-(u-\epsilon)^{+}}{\epsilon} d B_{u}\right]=\frac{H_{0}}{\sqrt{\epsilon}}\left[\int_{0}^{\epsilon} \frac{u-\epsilon}{\epsilon} d B_{u}\right]=H_{0} N_{\epsilon}
$$

where

$$
N_{\epsilon}=\int_{0}^{\epsilon} \frac{u-\epsilon}{\epsilon \sqrt{\epsilon}} d B_{u} .
$$

The r.v $N_{\epsilon}$ does not depend on $t$ anymore and is independent from $\mathcal{F}_{0}$. Moreover $N_{\epsilon}$ has a centered Gaussian distribution, with variance

$$
E\left(N_{\epsilon}^{2}\right)=\int_{0}^{\epsilon}\left(\frac{u}{\epsilon}-1\right)^{2} \frac{d u}{\epsilon}=\frac{1}{3} .
$$

Finally, $N_{\epsilon}$ follows the Gaussian law $\mathcal{N}(0, \sigma)$.

Note that $\Delta_{\epsilon}\left(H_{0}, 0\right)=0$. Consequently, the process $\left(\Delta_{\epsilon}\left(H_{0}, t\right)\right)_{t \geqslant 0}$ cannot converge in distribution as $\epsilon \rightarrow 0$.

\section{References}

[1] Blandine Bérard Bergery. Approximation du temps local et intégration par régularisation. PhD thesis, Nancy Université, 2004-2007.

[2] M. Gradinaru and I. Nourdin. Approximation at first and second order of $m$ order integrals of the fractional Brownian motion and of certain semimartingales. Electron. J. Probab., 8:no. 18, 26 pp. (electronic), 2003.

[3] A. Jakubowski, J. Mémin, and G. Pagès. Convergence en loi des suites d'intégrales stochastiques sur l'espace $\mathbf{D}^{1}$ de Skorokhod. Probab. Theory Related Fields, 81(1):111-137, 1989. 
[4] D. Revuz, and M. Yor. Continuous martingales and Brownian motion, volume 293 of Grundlehren der Mathematischen Wissenschaften [Fundamental Principles of Mathematical Sciences]. Springer-Verlag, Berlin, third edition, 1999.

[5] F. Russo and P. Vallois. The generalized covariation process and Itô formula. Stochastic Process. Appl., 59(1):81-104, 1995.

[6] F. Russo and P. Vallois. Itô formula for $C^{1}$-functions of semimartingales. Probab. Theory Related Fields, 104(1):27-41, 1996.

[7] F. Russo and P. Vallois. Stochastic calculus with respect to continuous finite quadratic variation processes. Stochastics Stochastics Rep., 70(1-2):1-40, 2000.

[8] F. Russo and P. Vallois. Elements of stochastic calculus via regularisation. In Séminaire de Probabilités, XXXX, Lecture Notes in Math. Springer, Berlin, 2006. 